\title{
Nurse supported early discharge had a readmission rate similar to conventional hospital care in patients with exacerbations of chronic obstructive pulmonary disease
}

\author{
Cotton MM, Bucknall CE, Dagg KD, et al. Early discharge for patients with exacerbations of chronic obstructive \\ pulmonary disease: a randomised controlled trial. Thorax 2000 Nov,55:902-6.
}

\section{QUESTION: How does early discharge with home respiratory nurse support compare with conventional hospital management for subsequent need for readmission in patients with acute exacerbations of chronic obstructive pulmonary disease (COPD)?}

Design

Randomised (allocation concealed), unblinded, controlled trial with 60 days of follow up.

Setting

A large university hospital in Glasgow, UK.

\section{Patients}

81 patients (mean age 67 y, 57\% women) who were admitted on an emergency basis with an acute exacerbation of COPD. Exclusion criteria were other medical conditions that required inpatient investigation or management, acidotic respiratory failure, nonresident status in Glasgow, homelessness, or lack of telephone access. 93\% of patients received their allocated intervention, but all patients were included in the intention to treat analysis.

Sources of funding: Alle and Hanburys Ltd, Greater Glasgow Health Board, and Glasgow Royal Infirmary University NHS Trust.

For correspondence: Dr R D Stevenson,

Department of Respiratory Medicine, Glasgow Royal Infirmary, Glasgow

G31 2ER, UK. Fax +44 (0)1412114932

\section{Intervention}

41 patients were allocated to early discharge, 36 of whom were sent home the next working day after recruitment. These patients had home visits by specialist respiratory nurses on the first morning after discharge and thereafter at intervals determined by the nurse. Home treatment was based on the practice developed by the Acute Respiratory Assessment Service. The nurse assessed progress and could adjust treatment after discussion with respiratory medical staff. The nurse did not prescribe, but could advise patients on the use of "as required" medication.

40 patients were allocated to conventional hospital care. Specialist respiratory consultation was only given if specifically requested.

\section{Main outcome measures}

Readmission rate, additional days in hospital, and 60 day mortality rate.

\section{Main results}

Analysis was by intention to treat. The mean number of days in hospital was 3.2 days for the nurse supported early discharge group and 6.1 days for the conventional hospital care group. Patients in the nurse supported early discharge group had a median duration of nurse follow up of 24 days and a median number of nurse home visits of 11. No differences existed between nurse supported early discharge and conventional hospital care for readmission rates $(29 \% v 30 \%)$, additional days in hospital (7.83 $v 8.75)$, or 60 day mortality rate $(2.4 \% v 5 \%)$.

\section{Conclusion}

Early discharge with respiratory nurse home visits was associated with similar readmission rates and subsequent days in hospital as conventional hospital care in patients with an acute exacerbation of chronic obstructive pulmonary disease.

\section{COMMENTARY}

With the increasing pressure to reduce hospital use, there is a need to identify patients who can be cared for at home rather than in hospital and to determine the necessary services required for effective home based care. These 2 studies by Cotton $e t$ al and Skwarska $e t$ al make an important contribution in both areas. Earlier studies evaluating the effects of home based care by outreach nurses for people with COPD have shown some improvement in mortality and quality of life, but no data exist on reductions in hospital usage. ${ }^{1}$ These 2 studies extend the literature by showing that a select group of patients with exacerbations of COPD in hospital can be discharged early with home based care. Results showed that hospital stay was reduced while maintaining comparable levels of safety and treatment success as those patients who were cared for in hospital. This evidence is strengthened by the randomised controlled trial designs.

Both studies identified patients with mild to moderate exacerbations of COPD and limited comorbidity as appropriate for early discharge service. In both studies, most patients admitted to hospital were not considered eligible for home care on medical grounds. Therefore, screening for the appropriateness of patients is critical to the safety and effectiveness of early discharge home based care. Consideration should be given to incorporating these study findings into the established guidelines for the management of COPD. ${ }^{2-4}$

Skwarska $e t$ al provided a more indepth outcome analysis, evaluating the effects on quality of life as measured by the Chronic Respiratory Questionnaire No difference was found between groups for quality of life. Although this is an important measure for the patient, neither this study nor the one by Cotton et al evaluated the effects on caregiver burden. Skwarska et al showed that general practitioners and patients with COPD found early discharge service acceptable, although satisfaction levels of the home based group were not compared with the usual care group. Future studies should examine if home based care shifts the burden of care from the healthcare system to the caregivers.

The cost to caregivers was not considered in the economic analysis by Skwarska et al. Although the study showed significant cost savings with supported discharge, only traditional health service costs and not those attributed to the caregivers or community health resources (other than general practitioner care) were measured.

The home based service in both studies involved home visits by nurses with specialised respiratory knowledge and expertise in providing home visiting, and consultation with respiratory specialists. Such home based care interventions will be of particular interest to those who are developing community based continued on next page 